\title{
Investigation
}

\section{Gene body methylation is under selection in Arabidopsis thaliana}

\author{
Aline Muyle*, Jeffrey Ross-Ibarra ${ }^{\dagger}$, Danelle K. Seymour ${ }^{\ddagger}$, Brandon S. Gaut ${ }^{*}$
}

5 *Ecology and Evolutionary Biology, UC Irvine, Irvine.

†Evolution and Ecology, UC Davis, Davis.

$\ddagger$ Botany \& Plant Sciences, UC Riverside, Riverside, United States.

Corresponding author: Aline Muyle, 321 Steinhaus Hall, University of California Irvine, CA 926972525 USA, +1 (949) 824-6006, amuyle@uci.edu

\begin{abstract}
In plants, mammals and insects, some genes are methylated in the CG dinucleotide context, a phenomenon called gene body methylation. It has been controversial whether this phenomenon has any functional role. Here, we took advantage of the availability of 876 leaf methylomes in Arabidopsis

15 thaliana to characterize the population frequency of methylation at the gene level and estimated the site-frequency spectrum of allelic states (epialleles). Using a population genetics model specifically designed for epigenetic data, we found that genes with ancestral gene body methylation are under significant selection to remain methylated. Conversely, all genes taken together were inferred to be under selection to be unmethylated. The estimated selection coefficients were small, similar to the

20 magnitude of selection acting on codon usage. We also estimated that $A$. thaliana is losing gene body methylation three-fold more rapidly than gaining it, which could be due to a recent reduction in the efficacy of selection after a switch to selfing. Finally, we investigated the potential function of gene
\end{abstract}


body methylation through its link with gene expression level. Across genes with polymorphic methylation states, the expression of gene body methylated alleles was consistently and significantly

25 higher than unmethylated alleles. Although it is difficult to disentangle genetic from epigenetic effects, our work suggests that gbM has a small but measurable effect on fitness, perhaps due to its association to a phenotype like gene expression.

\section{Introduction}

Cytosine DNA methylation is a type of epigenetic mark in which a methyl group is added to the $5^{\text {th }}$ carbon of cytosines. In plants, it can occur in three sequence contexts - CG, CHG and CHH (where $\mathrm{H}$ stands for $\mathrm{A}, \mathrm{T}$ or $\mathrm{C}$ ) - but levels and patterns of DNA methylation vary among genomic regions. In flowering plants, repetitive regions tend to be methylated in all three contexts, where methylation has a

35 well-established repressive function on transposable elements (TEs) and regulatory elements (Luo et al. 2018; Schmitz et al. 2019). In contrast, exons in plants, insects and mammals are sometimes methylated only in the CG context. This gene body methylation (gbM) can be found within moderately and constitutively expressed housekeeping genes (Zhang et al. 2006; Neri et al. 2017; Schmitz et al. 2019) and is linked to active transcription in plants (Zhang et al. 2006; Zilberman et al. 2007; Cokus et al. 2008; Lister et al. 2008). However, it is not yet clear if gbM has a function, because the study of mutants deprived of gbM has failed to reveal a clear effect on phenotype (Teixeira and Colot 2009; Bewick and Schmitz 2017; Zilberman 2017).

The mechanisms responsible for the establishment of gbM in plants have recently been clarified, due in large part to studies in Eutrema salsugineum, a close relative of Arabidopsis thaliana

45 that lacks both gbM and the CHROMOMETHYLASE 3 (CMT3) gene (Bewick et al. 2016). The CMT3 
protein has previously been shown to be involved in a self-reinforcing feedback loop: the histone mark H3K9me2 is recognized by CMT3 which then de novo methylates nearby cytosines in the CHG context and in turn leads to H3K9 methylation (Kawashima and Berger 2014). The deposition of CHG methylation typically suppresses transcription, but it is removed within transcribed genic regions by

50 INCREASED IN BONSAI METHYLATION 1 (IBM1) (Saze et al. 2008; Miura et al. 2009).

The critical role of CMT3 in gbM establishment is supported by the facts that not just one but two Brassicaceae species have independently lost CMT3 and both lack gbM (Bewick et al. 2016; Niederhuth et al. 2016). Moreover, transgenic reinsertion of CMT3 into E. salsugineum re-establishes genic methylation in all three contexts in a subset of genes that tend to be orthologous to gbM genes in

55 A. thaliana (Wendte et al. 2019). This subset of genes has been called 'CHG-gain' genes (Wendte et al. 2019), and remarkably, these genes remained methylated only in the CG context following the loss of the CMT3 transgene (Wendte et al. 2019). It remains unclear how CMT3 (and/or H3K9me2) is directed to a specific subset of genes for de novo DNA methylation and how these CHG-gain genes also become de novo methylated in the CG and CHH contexts (Wendte et al. 2019), but cmt3 mutants in A. thaliana 60 clearly demonstrate that CMT3 does not affect the maintenance of gbM once it is established (Stroud et al. 2013). Once CG methylation is established, it is maintained by METHYLTRANSFERASE 1 (MET1), which adds a methyl group on the symmetrical CG dinucleotide of a complementary DNA strand during cell duplication (Kawashima and Berger 2014). Maintenance by MET1 is an inherently error-prone process, as illustrated by epimutation accumulation in A. thaliana (Becker et al. 2011;

65 Schmitz et al. 2011; van der Graaf et al. 2015). The accumulation of these epimutations over time illustrates that CG methylation is heritable.

Although gbM is widespread across species and relatively common within a genome - it is found for example in $\sim 20 \%$ of A. thaliana genes (Takuno and Gaut 2012) - it remains unclear whether gbM is functionally relevant (Teixeira and Colot 2009; Bewick and Schmitz 2017; Zilberman 2017). 
70 The question of its potential function has focused on three interrelated hypotheses. The first is that gbM affects gene expression. This hypothesis is supported by the fact that gbM genes exhibit a positive correlation between methylation and expression levels across genes (Zhang et al. 2006; Zilberman et al. 2007; Takuno and Gaut 2012), suggesting either that gbM might cause higher expression or, conversely, that active transcription drives gbM (Teixeira and Colot 2009). However, further tests of

75 this association have led to contradictory results. For example, not all highly expressed genes have gbM in A. thaliana (Zhang et al. 2006; Zilberman et al. 2007), illustrating that any association is not absolute. The association has also been tested experimentally in epigenetic recombinant inbred lines (epiRILs) that were developed from the cross of a met1 mutant and wild-type (WT) A. thaliana, followed by eight generations of inbreeding (Reinders et al. 2009). The resulting epiRILs had a mosaic

80 methylome, with regions that have normal CG methylation derived from the WT parent and other regions derived from the met1 mutant that originally lacked gbM. Analysis of gene expression in these lines detected no significant changes in the met1 derived regions of epiRILs compared to orthologous WT regions (Bewick et al. 2016). Moreover, the epiRILs did not reestablish the original pattern of gbM after eight generations of epimutations (Bewick et al. 2016), suggesting that expression was not

85 sufficient to drive gbM reestablishment, at least not within a few generations. However, Zilberman et al. (2007) found that both methylated and unmethylated genes were upregulated in met1 mutants using microarray data, suggesting met1 methylation mutants may have unanticipated global expression effects that make them a poor system for studying the association between gbM and expression.

Another approach to test for associations between gbM and expression has been comparative 90 genomics, which has the advantage of integrating effects over evolutionary time. Here again the results have been inconsistent. For example, Bewick et al. (2016) and Bewick et al. (2019) found no effect of the loss of gbM on gene expression in E. salsugineum compared to A. thaliana. In contrast, Muyle and Gaut (2019) found a small but significant decrease in expression associated with genes that lost gbM in 
E. salsugineum, based on a reanalysis of the data from Bewick et al. (2016). In another effort, Takuno

95 et al. (2017) identified genes that changed methylation status between A. thaliana and Arabidopsis lyrata. They found a trend: genes that had gained gbM between species tended to also shift toward higher expression levels. Finally, Seymour and Gaut (2019) studied eight grass species and found that genes that were gbM in all eight species tended to have higher and less variable expression, although the effect is small. This last observation is consistent with previous observations that gbM is associated

100 with less variable gene expression both within and between species (Zilberman et al. 2008; ColemanDerr and Zilberman 2012; Steige et al. 2017; Takuno et al. 2017; Horvath et al. 2019; Seymour and Gaut 2019), suggesting it has a homeostatic effect on expression (Zilberman 2017).

In addition to a potential - but unresolved - association with gene expression, a second hypothesis of gbM function is that it prevents aberrant internal and/or antisense transcription (Tran et

105 al. 2005; Maunakea et al. 2010). Here again the evidence is unclear, because studies comparing gbM mutants to wild type mouse embryonic stem cells have been contradictory (Neri et al. 2017; Teissandier and Bourc’his 2017). In plants, Bewick et al (2016) found no evidence that gbM prevents antisense transcription in met1 derived regions of $A$. thaliana epiRILs compared to orthologous wild type regions. However, Choi et al (2020) has shown that gbM and histone H1 jointly suppress antisense 110 transcription in a comparison of met1,h1 double mutants to WT A. thaliana.

The third hypothesis is that gbM improves splicing fidelity and prevents intron retention. There is some evidence for this hypothesis, because the alteration of DNA methylation impacts alternative splicing in honey bee and mouse embryonic stem cells (Li-Byarlay et al. 2013; Yearim et al. 2015). Horvath et al. (2019) has found evidence to support this hypothesis by comparing gbM genes to 115 unmethylated genes in A. thaliana, but Bewick et al. (2016) found no evidence for this effect by comparing met1 epiRILs to wild type plants. Overall, the contradictory findings regarding the possible function of gbM suggests that its effects, if any, must be relatively small. 
While assays of the functional relevance of gbM have provided mixed results, evolutionary patterns of gbM have provided consistent but indirect evidence of its potential importance. Across plant

120 species, gbM genes are generally longer, enriched for housekeeping and other important functions and evolve more slowly than unmethylated genes (Takuno and Gaut 2012, 2013; Takuno et al. 2017; Seymour and Gaut 2019). Moreover, comparative analyses have shown that gbM is conserved for orthologous genes between species as distantly related as ferns and angiosperms (Takuno and Gaut 2013; Seymour et al. 2014; Takuno et al. 2016; Niederhuth et al. 2016; Seymour and Gaut 2019). This

125 last characteristic of gbM is surprising because DNA methylation is mutagenic and elevates $\mathrm{C}$ to $\mathrm{T}$ substitutions (Bird 1980). Hence, the conservation of gbM over millions of years suggests that the mutagenic feature of methylation is counterbalanced by an advantageous effect that acts to maintain gbM in specific genes (Zilberman 2017). However, another possible explanation for the strong conservation of gbM within a specific set of genes is that de novo methylation biases, such as those that

130 target the CHG-gain genes of E. salsugineum (Wendte et al. 2019), have been conserved across species over vast periods of evolutionary time.

Clearly several questions about gbM function and evolution remain unresolved. Here we move away from experiments and comparative studies and employ population genetic approaches to study gbM. Thus far, the tools of population genetics have been applied to epigenetic phenomena in only a handful

135 of studies. For example, van der Graaf et al. (2015) found similar epimutation rates between $A$. thaliana populations and $>31$ generations of epimutation accumulation lines, suggesting that selection has not impacted global patterns of CG methylation diversity in that species. They nonetheless argued, based on the rate of epimutation events, that selection of epiallelic states could be an important process. Wang and Fan (2014) developed a modification of Tajima’s D for application to methylation data and

140 used it to demonstrate that new genes have an excess of rare epialleles, which they interpreted was consistent with directional selection on an epigenetic state. Two other studies have used site frequency 
spectra (SFS) to test for selection on methylation data. In the first, Vidalis et al. (2016) estimated the SFS of cytosine sites within genes of a sample of 92 A. thaliana individuals, but they did not detect a deviation from neutrality. More recently, studies have hinted at selection on methylation, because an

145 SFS analysis at the level of 100bp regions detected weak but significant selection on methylation levels (Xu et al. 2020) and because germline promoter methylation was inferred to be deleterious in humans (Boukas et al. 2020).

Here we extend the SFS approach to data from the 1001 methylomes project in A. thaliana (Kawakatsu et al. 2016), to test two features of gbM. The first is whether there is evidence that gbM is subject to

150 selection. To do so, we focus on the methylation state of genes, rather than individual sites. We focus on genes because previous work has shown that methylation is evolutionary conserved at the level of genes and not within individual sites, suggesting that the methylation state of a gene region could be the unit under selection (Takuno and Gaut 2013). Consistent with this hypothesis, Vidalis et al. (2016) found no evidence of selection at the cytosine level. The second is that we provide an intraspecific test

155 of the association of gbM and gene expression by comparing the methylation state of alleles to their level and variability in expression. By harnessing the power of an extensive $A$. thaliana data set, we uncover new information on the evolutionary forces that may act on epigenetic phenomena and the potential functional significance of gbM.

\section{Materials and Methods}

\section{Datasets}

Methylation and expression files for A. thaliana from Kawakatsu et al. (2016) were downloaded from GEO (accessions GSE43857, GSE80744, GSE54292, GSE43858, GSE54680). The 
165 files consisted of tables with one line per cytosine showing the number of methylated and unmethylated bisulfite sequencing (BS-seq) reads for each methylome, and tables with one line per gene showing the number of reads mapping for each transcriptome. The dataset included 1211 samples sequenced by BSseq and 1195 by RNA-seq. More precisely, 927 A. thaliana were grown at $22^{\circ} \mathrm{C}$ and their methylomes were sequenced by BS-seq at the SALK Institute (Kawakatsu et al. 2016), of which 876 came from

170 leaves and 51 from flower buds (with only a partial overlap in accessions between the two tissues). 144 samples had their leaf transcriptome profiled with the SOLiD system (Schmitz et al. 2013), and 728 samples had their leaf transcriptome sequenced by Illumina RNA-seq (Kawakatsu et al. 2016). Swedish accessions had their leaf BS-seq data generated at the Gregor Mendel Institute (GMI) (Dubin et al. 2015). These included 152 accessions that were grown at $10^{\circ} \mathrm{C}$ and another 121 accessions grown

175 at $16^{\circ} \mathrm{C}$. Some accessions had replicates sequenced, resulting in a total of 284 methylomes from GMI. These had corresponding leaf transcriptome data from 160 accessions grown at $10^{\circ} \mathrm{C}$ and from 163 accessions grown at $16^{\circ} \mathrm{C}$, for a total of 323 samples sequenced by Illumina RNA-seq (Dubin et al. 2015). However, we detected a strong Institute-of-origin effect in the data (see Results), leading us to focus most of our analyses on leaf data from the Salk Institute (876 accessions).

180 To have outgroup data, we retrieved A. lyrata MN47 and Capsella rubella MTE 10 day old seedling shoot methylation files (Seymour et al. 2014). For each species, two replicates grown at $23^{\circ} \mathrm{C}$ were used.

\section{Inference of cytosine methylation}


methylation using the same method. Briefly, methylation was inferred for each site by performing a binomial test on the number of methylated and unmethylated reads, while taking into account the non-

190 conversion rate (Lister et al. 2008). For the GMI data, the average non-conversion rate of 0.0041 was used for all samples (Dubin et al. 2015). P-values were corrected for multiple tests using Benjamini and Hochberg correction. Sites with $\leq 2$ reads were considered as unmethylated, and sites with a corrected p-value under 0.001 were considered to be methylated.

\section{Inference of gene body methylation}

For each gene, the methylation state was inferred using data from coding sequences (CDS), which included exons but excluded both untranslated terminal regions and introns. We used the annotation of the longest transcript to define the CDS. For each accession separately, we computed an expected methylation rate for each context (CG, $\mathrm{CHG}, \mathrm{CHH})$ across all CDSs annotated in the genome,

200 and we used binomial tests to assess whether gene CDSs had a significantly higher proportion of methylated cytosines than the genome-wide background level of CDS methylation (Takuno and Gaut 2012). This was performed for each accession and cytosine context separately. P-values were corrected for multiple tests using the Benjamini and Hochberg correction for each accession separately.

Given the binomial results, a gene within an accession was inferred to be gene body methylated (gbM) if it had more than 20 CG sites and if CG methylation was significantly higher than the background (one-sided binomial p-value lower than 0.05) and CHG and CHH methylation were not significantly higher than the background (one-sided p-values higher than 0.05). A gene was inferred to be CHG methylated if it had more than 20 CHG sites and if CHG methylation was higher than the background (one-sided p-value lower than 0.05) and $\mathrm{CHH}$ methylation was not significantly higher

210 than the background (one-sided p-values higher than 0.05). CHG methylated genes also tended to be CG methylated, but CG methylation was not required in our categorization. A gene was inferred to be 
CHH methylated if it had more than $20 \mathrm{CHH}$ sites and if $\mathrm{CHH}$ methylation was higher than the background (one-sided p-value lower than 0.05). CHH methylated genes also tend to be CG and CHG methylated. Finally, a gene was inferred to be unmethylated (UM) if it had more than 20 CG sites and

215 if CG, CHG and CHH methylation were not significantly higher than the background (one-sided pvalue higher than 0.05). In any other case, the gene methylation state was not inferred. Altogether, by applying this approach, we identified the frequency of methylation states across alleles among 1211 accessions and for $\sim 27,000$ genes.

\title{
220 Inference of ancestral methylation state
}

For each gene, the ancestral methylation state in A. thaliana was inferred using methylation data from A. lyrata and C. rubella. To this end, we used the CoGe tool SynMap3D (Lyons and Freeling 2008) to infer orthologous syntelogs among A. thaliana, A. lyrata and C. rubella. We differentiated between orthologs and out-paralogs (paralogs caused by duplications that predate speciation) using

225 pairwise dS values between syntelogs. Based on the distribution of dS values (Supplementary Figure S1), $\log 10(\mathrm{dS})$ values were filtered to be lower than -0.39 for all species pairwise comparisons, which is equivalent to dS values lower than 0.407 . After this filtering, 14,718 orthologous syntelogs were identified among the three species.

Two shoot replicates grown at $23^{\circ} \mathrm{C}$ were available for each outgroup species (A. lyrata and $\mathrm{C}$.

230 rubella) (Seymour et al. 2014). For every gene, the ancestral methylation state was inferred as the shared state between the two outgroups and their replicates. If the two replicates of a species had different methylation states for a gene, or if the gene had different methylation states between A. lyrata and C. rubella, we excluded it from analyses as having an ambiguous ancestral state.

\section{Inference of genes undergoing CG methylation epimutations}

\author{
Page 10 of 38
}


We also investigated the set of CHG-gain genes from E. salsugineum CMT3 overexpressing transgenic lines by retrieving the list of 8,704 CHG-gain genes from Wendte et al. (2019). The best blast hit - as provided in the genome reference - was used to infer the ortholog in A. thaliana for 8,025 of these CHG-gain genes.

\title{
Site frequency spectrum (SFS)
}

The unfolded SFS was drawn for two gene methylation states, gbM and UM. mCHG and $\mathrm{mCHH}$ states were excluded from the SFS (see Supplementary Figure S2 for the distribution of the proportion of $\mathrm{mCHG}$ and $\mathrm{mCHH}$ accessions across all genes). Genes were included in the SFS only

245 when methylation status could be determined in 200 or more accessions, and genes that had over $70 \%$ of accessions with mCHG or mCHH methylation state were discarded as possibly being pseudogenes or misannotated TEs.

The number of accessions with an inferred methylation state $n$ varied among genes due to missing data, so that the site frequency spectrum sample size varied among genes. To cope with this

250 missing data, we defined $n$ ', the minimum required number of accessions with characterized methylation, and applied a hypergeometric projection of the observed SFS into a subsample of size n'=200. Genes sampled in less than $n$ ' accessions were discarded. The frequency of the derived allele in the reduced sample follows a hypergeometric distribution. Given $k$ the frequency of the derived allele in the original sample of size $n$, the probability that $i$ copies were observed in the reduced sample

255 of size $n^{\prime}$ is (Hernandez et al. 2007):

$P\left(\frac{i}{n^{\prime}} \mid \frac{k}{n}\right)=\frac{C_{k}^{i} C_{n-k}^{n^{\prime}-i}}{C_{n}^{n^{\prime}}}$

\section{Estimation of selection using the site frequency spectrum}

\author{
Page 11 of 38
}


Given the SFS, we estimated the strength of selection acting on methylation variants using the

260 model of Charlesworth and Jain (2014). The model was designed to characterize the evolutionary forces acting on epigenetic markers, which evolve at much higher rates than DNA sequences when single sites are considered (Becker et al. 2011; Schmitz et al. 2011). We adapted the model for application to our biological question of whether selection acts on gene methylation states. Genes can either be gbM or UM, with $\mu$ and $v$ the mutation rates from one state to the other:

265

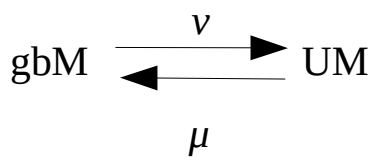

The model assumes a randomly mating diploid population of constant effective population size $N_{e}$ which is at mutation-selection equilibrium. The model further assumes that alleles 270 are semi-dominant and that sites are independent. We estimated $N_{e}$ using available polymorphism measures in A. thaliana (Alonso-Blanco et al. 2016): 10,707,430 total SNPs were detected in 1135 genomes of size $135 \mathrm{Mb}$, resulting in a Watterson theta $\theta_{w}=0.00955$ (Charlesworth and Charlesworth 2010). Using a mutation rate $\mu=7 \cdot 10^{-9}$ (Ossowski et al. 2010) and $\theta_{w}=4 N_{e} \mu$, we estimated $N_{e} \approx 341,000$. These values were similar to previous diversity measurements in $A$. thaliana, where intronic $\theta_{w}$ was 275 estimated to be 0.0082 (Nordborg et al. 2005), but the actual $\theta_{w}$ may be higher due to biases in its estimation (Korunes and Samuk 2020).

If the UM state is advantageous over the gbM state, the probability that a sample of $n$ individuals segregates for $k$ UM variants and ( $n-k)$ gbM variants at a given gene is (Charlesworth and Jain 2014):

$280 p(k)=\left(\begin{array}{l}n \\ k\end{array}\right) \frac{F_{1}(\beta+k, \alpha+\beta+n, \gamma)(\beta)_{k}(\alpha)_{n-k}}{F_{1}(\beta, \alpha+\beta+n, \gamma)(\alpha+\beta)_{n}}$ 
Where $F_{1}$ is the confluent hypergeometric function, $(x)_{n}$ is Pochhammer's symbol, $\alpha=4 N_{e} \mu$, $\beta=4 N_{e} v$ and $\gamma=4 N_{e} S_{U M}$ with $s_{U M}$ the selective advantage of the UM methylation state over gbM. The model can easily be adapted to a case where the gbM state is advantageous over the UM state by switching $\alpha$ and $\beta$ in equation (3) and defining $s_{g b M}$ the selective advantage of the gbM state.

$$
\text { The likelihood of the model is: }
$$

$L=\prod_{k=0}^{n} p(k)^{d_{k}}$

Where $d_{k}$ is he number of genes observed with $k$ UM accessions and (n-k) gbM accessions.

Parameters of the model $\mu, v$ and $S_{U M}$ (or $s_{g b M}$ ) were estimated using a Markov Chain Monte Carlo (MCMC) random walk with 100,000 generations as in Xu et al. (2020). The first 25\% of MCMC

290 generations were removed as burn-in. Parameters were sampled every 100 generations, providing around 750 samples for the posterior distributions of parameters. The lambda parameters for scale proposal distribution were adjusted to obtain parameter acceptance rates between $20 \%$ and $70 \%$. Both segregating and fixed sites of the SFS were used in the model. Final parameter values were obtained from the mean of the posterior distribution and the credible interval from the $95 \%$ margins of the

295 posterior distribution. We ran the algorithm three times with random starting points to ensure that the global maximum was found. In order to test whether selection acting on the UM or the gbM state was significant, we compared the previous full model to a reduced model where the selection coefficient was fixed to zero using a likelihood ratio test with degree of freedom 1 . For each run, the expected SFS (using inferred parameter values from the best model) was compared to the observed SFS using a Pearson's $\chi$-square test in $\mathrm{R}$.

\section{Statistical study of the link between gbM and gene expression level}


We measured the effect of gbM on expression level using the Salk Institute leaf dataset, which consisted of 679 accessions with both leaf methylation data and leaf expression data in the form of raw

305 RNA-seq read counts. This number of accessions differed from the previous 876 Salk accessions used for the SFS analysis due to missing leaf expression data for some accessions. We constructed a linear model with mixed effects (equation 5) to examine the data, which was run with the R package lme4 (Bates et al. 2015). We did not normalize gene expression and used raw read numbers, but results were equivalent when using normalized read numbers as provided in GEO expression files. The aim of the model was to test, within each gene, for an association between a change in gene methylation state and gene expression across A. thaliana samples. To account for expression variability among genes, the model incorporated a random gene effect (see equation 5). The random gene effect captures variability in gene expression due to average differences among genes. We also defined a fixed effect called gene methylation state (equation 5), which consists of the states described above (e.g., gbM, mCHG, mCHH

315 and UM) and applies to each gene epiallelic state within each accession. Significance for the fixed effect was determined by comparing the fit of the full model to a nested model without the fixed effect, using the anova function in R. Expression level was measured as raw read counts and log transformed. The R package lsmeans (Lenth 2016) was used to estimate pairwise differences between each pair of methylation states (i.e. gbM versus UM, UM versus mCHG etc.).

Our linear model can be expressed as:

$\log ($ Gene Expression + 1) gene methylation state + (1|Gene)

We also developed two linear mixed-effects models to investigate the potential relationship between genetic and epigenetic states of alleles. The model included the number of CG dinucleotides (\#CG) and the epiallelic methylation state, as fixed effects, and the random gene effect:

$$
\begin{aligned}
& \text { \#CG gene methylation state + (1|Gene) } \\
& \log (\text { Gene Expression + 1) \#CG + gene methylation state + (1|Gene) }
\end{aligned}
$$




\section{Results}

\section{Detection of selection acting on gene methylation level}

We used publicly available methylation datasets (Seymour et al. 2014; Kawakatsu et al. 2016) to infer the methylation state of genes in A. thaliana accessions and two closely related outgroups. We first recognized that BS-seq sequencing of the A. thaliana 1001 methylomes was carried out by two research Institutes (Salk and GMI), and so we compared methylation patterns and levels between the

335 two institutes. We found that the global rate of $\mathrm{CHH}$ methylation was significantly higher in accessions sequenced by GMI (2.3\%) compared to the Salk Institute (0.28\%, Supplementary Figure S3), regardless of the geographic origin of accessions (Supplementary Figure S4). This heterogeneity in the raw data had the potential to impact downstream gene methylation inferences (Supplementary Figure S5). We therefore focused on a single source - i.e, the Salk data - because it had the highest number of samples (927 methylomes). For similar reasons, we also focused only on methylome data from a single tissue (leaf), leading to total analysis sample of 876 accessions.

Given the data, we inferred the gbM status for each gene in each individual separately to calculate the unfolded SFS for two gene methylation states - gbM and UM - after downsampling to 200 accessions (see Material and Methods for details). Altogether, we plotted the SFS based on 23,868

345 genes and found that, after downsampling to 200 accessions, most genes were fixed for the UM methylation state in $A$. thaliana wild populations (11,901 genes), but there was also a subset of 983 genes fixed for gbM alleles (Figure 1.A). Given the inferred SFS, we applied the model of Charlesworth and Jain (2014) to infer the selection coefficient, $s$ in a model where the UM state is advantageous and in another model for which the gbM state is advantageous. Based on all 23,868 genes 
350 together, we found that the model that best fit the data was one where the UM state is advantageous $\left(s_{U M}=8.68 .10^{-8}\right)$, with a $p$-value of 0.0086 based on comparing the two models with selection to a model without selection (Table 1). Note that the expected SFS based on estimated parameters fit the observed SFS quite well (Figure 1.A), with no significant difference between them (Pearson's $\chi$-squared test $\mathrm{p}=0.343)$.
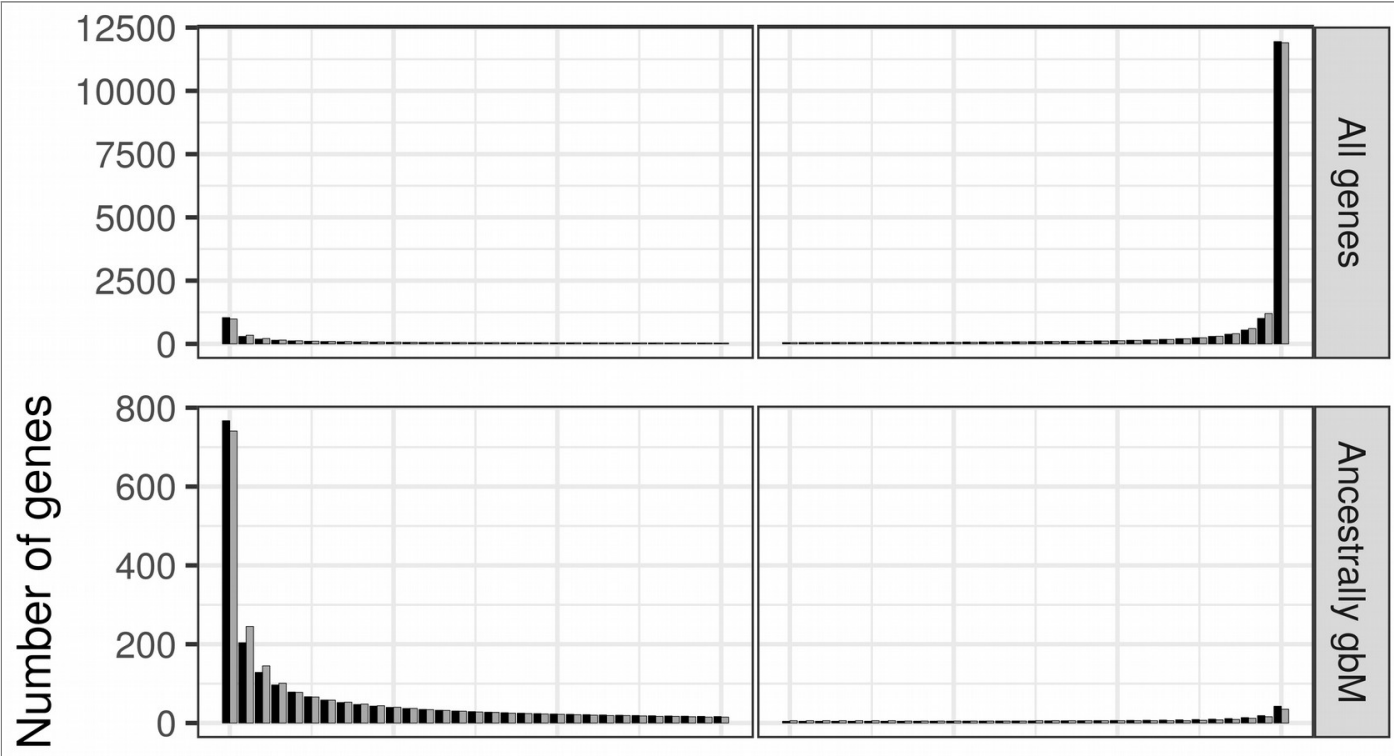

SFS type
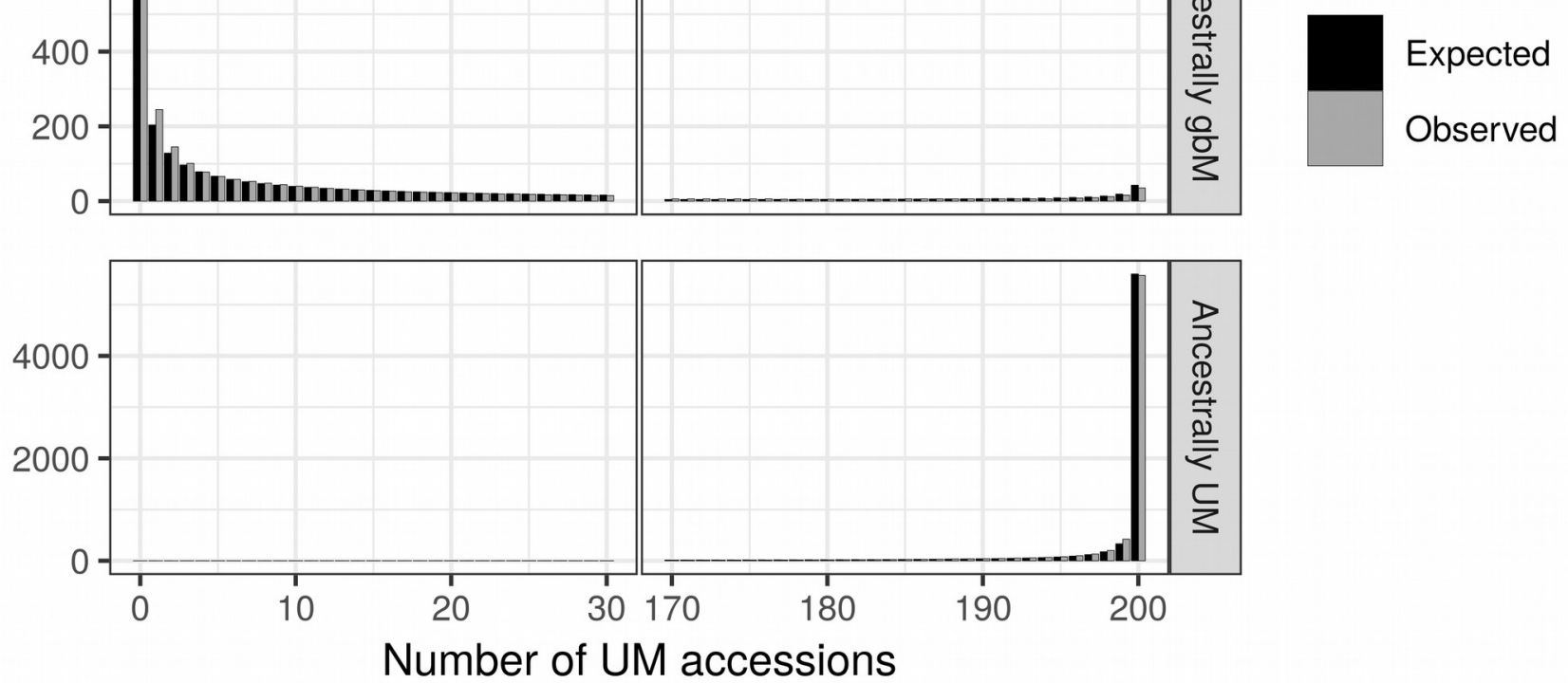

Figure 1: Expected and Observed Site Frequency Spectra (SFS) of gene body methylation. The x-axis provides the number of UM accessions, out of a sample of 200. For these data, accessions that are not UM are gbM, meaning that genes with 200 UM accessions are fixed for the UM state in $A$. thaliana and genes with 0 UM accessions are fixed for the gbM state. The number of genes is provided on the y-axis. A. All genes $(23,868$ genes), B. ancestrally gbM genes (3,239 genes) and C. ancestrally UM genes (7,780 genes). For visualization 
purposes, a gap was introduced in the x-axis. The expected SFS were drawn using the parameters estimated by the mcmc, using the best model in Table 1. All three expected SFS fit the observed SFS well and did not differ significantly from the observed distribution (Pearson's $\chi$-squared test $\mathrm{p}>0.4$ ).

Our SFS illustrates that gbM is markedly bi-modal, which is consistent with the fact that gbM is associated with a finite but conserved set of orthologous genes across angiosperms (Takuno et al. 2016). It seems reasonable to presume, then, that genes that are evolutionary conserved as gbM may be under different selection regimes than those that are evolutionary conserved as UM. Accordingly, we 370 repeated analyses after splitting ancestrally gbM and UM genes (Figures 1.B and 1.C). To infer the ancestral state, we used two outgroups (A. lyrata and C. rubella), identified syntelogs for 14,718 genes among the three species, and then inferred the ancestral methylation state by parsimony when both outgroups and their replicates had the same methylation state. After excluding 3,699 genes for either missing methylation state inferences or for having ambiguous ancestral state, we applied the model to a 375 set of 3,239 genes that were inferred to be ancestrally gbM, estimating a small $\left(s_{g b M}=1.08 .10^{-6}\right)$ but highly significant $\left(p=2.77 .10^{-17}\right.$ ) selection coefficient (Table 1 ). This result implies that there is weak but detectable selection to maintain methylated alleles within genes that are ancestrally gbM. In contrast, 7,780 ancestrally UM genes were estimated to be under no selection to retain UM nor gbM alleles in A. thaliana wild populations $\left(s_{U M}=s_{g b M}=0\right.$, Table 1$)$. 


\begin{tabular}{|c|c|c|c|c|c|}
\hline & & $\begin{array}{c}\text { Gene } \\
\text { number }\end{array}$ & Best model & p-value & Selection coefficient $s$ \\
\hline \multicolumn{2}{|c|}{ All genes } & 23,868 & $\begin{array}{c}\text { UM state } \\
\text { advantageous }\end{array}$ & 0.0086 & $\begin{array}{c}S_{U M}=8.68 .10^{-8} \\
\left(3.56 .10^{-8}: 1.46 .10^{-7}\right)\end{array}$ \\
\hline \multicolumn{2}{|c|}{ Ancestrally gbM genes } & 3,239 & $\begin{array}{c}\text { gbM state } \\
\text { advantageous }\end{array}$ & $2.77 .10^{-17}$ & $\begin{array}{c}S_{g b M}=1.08 .10^{-6} \\
\left(8.84 .10^{-7}: 1.28 .10^{-6}\right)\end{array}$ \\
\hline \multicolumn{2}{|c|}{ Ancestrally UM genes } & 7,780 & $\begin{array}{c}\text { no selection on gene } \\
\text { methylation }\end{array}$ & 1 & $s_{U M}=s_{g b M}=0$ \\
\hline \multicolumn{2}{|c|}{ CHG-gain orthologs } & 7,727 & $\begin{array}{c}\text { gbM state } \\
\text { advantageous }\end{array}$ & 0.00022 & $\begin{array}{c}S_{g b M}=1.73 .10^{-7} \\
\left(7.24 .10^{-8}: 2.62 .10^{-7}\right)\end{array}$ \\
\hline \multicolumn{2}{|c|}{ Non-CHG-gain orthologs } & 16,141 & $\begin{array}{c}\text { UM state } \\
\text { advantageous }\end{array}$ & $8.16 .10^{-11}$ & $\begin{array}{c}S_{U M}=3.12 .10^{-7} \\
\left(2.28 .10^{-7}: 3.92 .10^{-7}\right)\end{array}$ \\
\hline \multirow{2}{*}{$\begin{array}{l}\text { Ancestrally } \\
\text { gbM }\end{array}$} & $\begin{array}{l}\text { CHG-gain } \\
\text { orthologs }\end{array}$ & 1988 & $\begin{array}{c}\text { gbM state } \\
\text { advantageous }\end{array}$ & $1.19 .10^{-10}$ & $\begin{array}{c}S_{g b M}=1.16 .10^{-6} \\
\left(9.14 .10^{-7}: 1.39 .10^{-6}\right)\end{array}$ \\
\hline & $\begin{array}{c}\text { non-CHG-gain } \\
\text { orthologs }\end{array}$ & 1251 & $\begin{array}{c}\text { gbM state } \\
\text { advantageous }\end{array}$ & $2.58 .10^{-9}$ & $\begin{array}{c}S_{g b M}=1.36 .10^{-6} \\
\left(1.07 .10^{-6}: 1.66 .10^{-6}\right)\end{array}$ \\
\hline \multirow{2}{*}{$\begin{array}{c}\text { Ancestrally } \\
\text { UM }\end{array}$} & $\begin{array}{l}\text { CHG-gain } \\
\text { orthologs }\end{array}$ & 2274 & $\begin{array}{c}\text { no selection on gene } \\
\text { methylation }\end{array}$ & 1 & $s_{U M}=s_{g b M}=0$ \\
\hline & $\begin{array}{c}\text { non-CHG-gain } \\
\text { orthologs }\end{array}$ & 5506 & $\begin{array}{c}\text { no selection on gene } \\
\text { methylation }\end{array}$ & 1 & $s_{U M}=s_{g b M}=0$ \\
\hline
\end{tabular}

Table 1: Estimation of selection acting on gene methylation state. A likelihood-based approach was used to infer the best model to explain the SFS (see Materials and Methods for details). The p-value shows the result of the likelihood ratio test between the model with selection and the neutral model $(s=0)$. The estimated selection coefficient $s$ is shown with a 95\% credible interval in parenthesis. Details on inferred values of other parameters of the model can be found in Supplementary Table S1. These results come from one MCMC run and are 
equivalent to results obtained from two independent runs with random parameter initiation values (Supplementary Table S1).

We have mentioned both that there is no gbM in E. salsugineum due to the loss of CMT3 390 (Bewick et al. 2016) and that complementation of E. salsugineum with a functional copy of $A$. thaliana CMT3 leads to the accumulation of DNA methylation in 'CHG-gain' genes (Wendte et al. 2019). These results suggest that DNA methylation epimutation rates are not homogeneous among genes, which could be problematic for our model. We therefore repeated the previous analyses separately for CHG-gain and for non-CHG-gain genes in A. thaliana, based on identifying the orthologs of CHG-gain genes from E. salsugineum (Materials and Methods). We found that 7,727 CHG-gain genes were under significant selection for retaining the gbM state (Table 1), again implying that selection acts on gene methylation state. On the other hand, the set of 16,141 no-CHG-gain genes were estimated to be under selection to retain UM status. The model also estimates epimutation rates; consistent with implications of the E. salsugineum experiment (Wendte et al. 2019), we found that the mutation rate $\mu$ from the UM state to the gbM state was $\sim 1.77$ times higher in CHG-gain compared to no-CHG-gain genes. In contrast, the epimutation rate $v$ from gbM to UM was $\sim 0.79$ lower in CHG-gain compared to no-CHG-gain genes (Supplementary Table 1).

Finally, we repeated the analyses after splitting CHG-gain and non-CHG-gain genes into ancestrally gbM and ancestrally UM genes, because we have shown that the SFS of both features

405 (methylation state and CHG-gain state) suggest ongoing selection. Ancestrally gbM genes were under significant selection to remain gbM, regardless of whether they were targeted by additional methylation epimutations (CHG-gain) or not (non-CHG-gain, Table 1). However, ancestrally UM genes were under no significant selection to remain UM or become gbM (Table 1). Our results were therefore confirmed 
after splitting the dataset into sets of genes with putatively homogeneous epimutation rates; overall they

410 provide evidence that the methylation state of alleles is associated with natural selection.

\section{Effect of gene methylation state on gene expression level in A. thaliana wild populations}

E. salsugineum lacks gbM due to the loss of CMT3 (Bewick et al. 2016), but there has been some debate about the effects of this gbM loss on gene expression. Bewick et al (2016) found no effect,

415 a result upheld by later analyses (Bewick et al. 2019). However, using different statistical approaches, Muyle and Gaut (2019) found evidence for a small but significant decrease in expression level for $E$. salsugineum genes that had lost gbM relative to the same gbM genes in $A$. thaliana. We further investigated the possible association between gbM and gene expression by analyzing expression levels from the A. thaliana 1001 methylome data. To make this assessment, we focused on leaf expression

420 and methylation data from the Salk Institute for 679 accessions and 23,261 genes with polymorphic methylation states (i.e., genes fixed for a given methylation state were removed from consideration). The availability of these data permitted a test of whether epiallelic methylation states are associated with differences in expression.

We analyzed the data using a linear model with mixed effects (equation 5, Materials and

425 Methods). The model was written to measure within gene expression variation, and then test for a significant effect of methylation state across all genes. This approach is possible due to polymorphisms in gene epiallelic states (or epialleles) among accessions. Treating genes as random effects and epiallele state (gbM, UM, mCHG or $\mathrm{mCHH}$ ) as a fixed effect, we found that epiallele methylation state had a significant effect on gene expression level $\left(\chi^{2}=19,300\right.$ and $p$-value $=0$ when comparing a linear model

430 with and without gene methylation state effect). We repeated these analyses between pairs of methylation states, comparing along an expected hierarchy of expression levels defined as gbM > UM $>$ mCHG $>$ mCHH. Our results confirmed that expected hierarchy, because gene expression in 
accessions that had the gbM epiallelic state was significantly higher than for accessions that had the UM epiallelic state for that same gene, globally across all genes (Figure 2). Similarly, we found that 435 UM alleles had higher expression than mCHG alleles and that mCHG alleles were more highly expressed that $\mathrm{mCHH}$ alleles (Table 2). Altogether, these results show that within a gene, an accession with the gbM epiallelic state is consistently associated with the highest gene expression level, while the $\mathrm{mCHH}$ state is consistently associated with the lowest gene expression level. However, the estimated differences in expression levels were very small (0.0563 log read count difference on average between gbM and UM methylation states, Table 2, which is equivalent to 1.058 raw read count difference on average). It is important to note that linear models can detect small mean differences as significant so long as those differences are prevalent across the entire dataset.

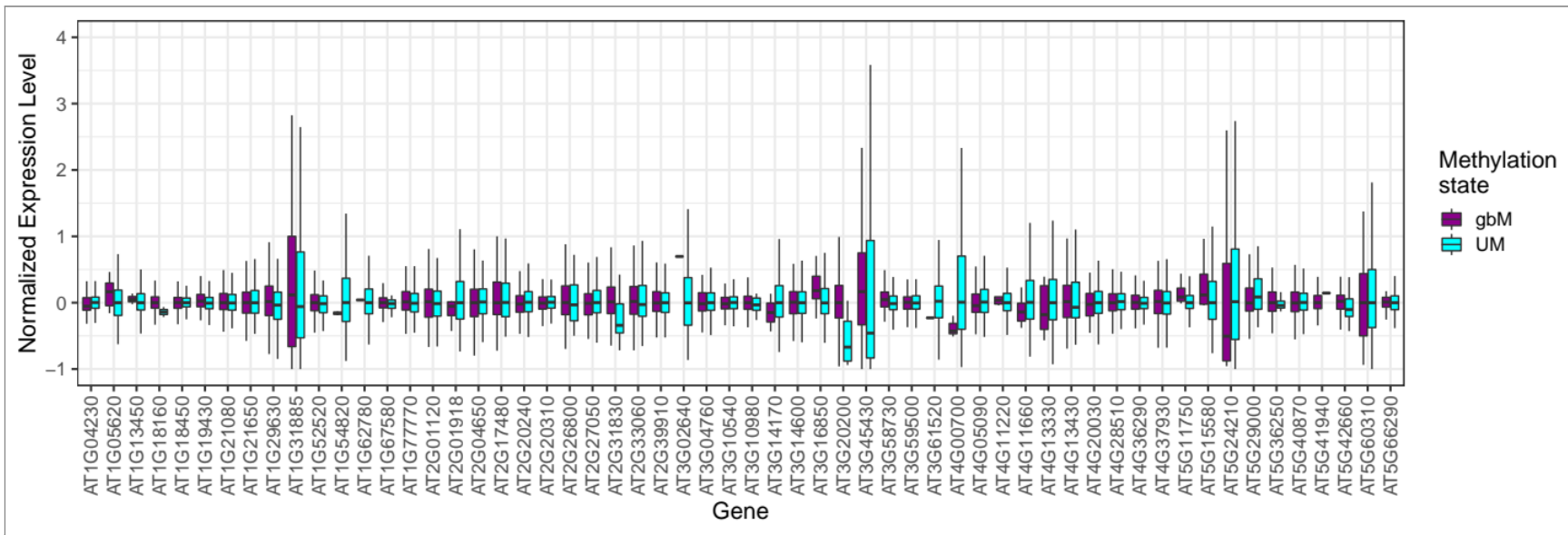

445 Figure 2: Normalized expression levels in a random set of genes with both UM and gbM epialleles. To make this figure, a random set of 100 genes was selected and only genes with both UM and gbM epialleles were retained. Genes with median read number under 10 were also excluded, leaving 57 genes. For each gene, the box plots indicate normalized expression levels for gbM and UM epialleles, with the lines representing the medians and the width of the boxplot the $1^{\text {st }}$ and $3^{\text {rd }}$ quartile. The figure shows that accessions that have a gbM epiallele 450 tend to have a higher median normalized expression compared to accessions that have a UM epiallele, because 34 out of 57 genes in the represented random gene set have higher median expression levels for gbM epialleles. 


\begin{abstract}
Although the differences are small and some genes show the opposite pattern, the overall effect is significant in a linear model across genes (see text for details). For each accession, normalized expression level was computed as follow: (accession normalized read number - median gene normalized read number) / median gene normalized read number.
\end{abstract}

In order to further test the robustness of our results, we performed two additional analyses. First, to confirm that the results were not an artifact of the linear model, we reran the model after randomly permuting methylation states without replacement among accessions and genes. These permutations removed associations between methylation states of an allele and their expression, and hence we did not expect to detect significant effects with permuted data. We ran the model on 1001 permuted datasets. As expected, the correlation between gene methylation state and expression was significant at $\alpha=0.05$ only $\sim 5.0 \%$ of the time, because we detected significance in 54 of 1001 permutations (Figure 3). What is more, the $p$-value obtained from the real dataset was more than 8 465 orders of magnitude lower than the lowest $p$-value obtained on any of the permuted datasets. These permutation results illustrate both that the model is well-behaved and that the observed data are strongly unexpected under a null hypothesis in which methylation and expression are not linked (Figure 3). Second, we compared expression levels between UM and gbM alleles within single genes for the 11,613 genes that had at least one UM accession and one gbM accession. We found accessions with 470 gbM epialleles had a higher median expression level than accessions with the UM state for 6,122 out of 11,613 (or $52.72 \%$ of genes). This proportion represents a significant deviation (binomial test, twosided, $p$-value $5.10^{-9}$ ) from the the expected value of $50 \%$ under the null hypothesis that epiallelic state does not affect expression. 


\begin{tabular}{|c|c|c|c|c|}
\hline Contrast & Estimate & Standard Error & z-ratio & p-value \\
\hline gbM - UM & 0.0563 & 0.0011 & 51.56 & 0 \\
\hline UM - mCHG & 0.1093 & 0.0033 & 33.13 & 0 \\
\hline mCHG - mCHH & 0.2275 & 0.00359 & 63.33 & 0 \\
\hline
\end{tabular}

Table 2: Pairwise comparison of the effect of gene methylation state on gene expression level in A. thaliana

1001 methylome data. A generalized linear model with mixed effects was used to estimate the effect of gene methylation state on gene expression (see Materials and Methods for details). Gene expression was measured as

480 raw read counts and log transformed, but the results were equivalent when performed on normalized read counts.

The table shows the average differences in log expression levels between pairs of methylation states (estimates) and their associated standard error, t-ratio and p-value after correction for multiple tests. For example, the gbM state is consistently associated with a 0.0563 higher log read count compared to the UM state, on average across all genes and accessions.

These data also present the opportunity to test the homeostasis hypothesis, which posits that gbM acts to stabilize gene expression (Zilberman 2017). Under this hypothesis, we expect the coefficient of variation to be lower across gbM epialleles than for UM epialleles. To test the hypothesis, we focused on the 10,327 genes that harbored at least two accessions of both UM and gbM epiallelic states in the

490 population and then controlled for sample size differences by randomly sampling the same number of accessions for both epialleles within each gene. We found that the gbM state had a significantly lower coefficient of variation than the UM state (one-sided Wilcoxon paired signed rank test $\mathrm{p}=4.77 .10^{-6}$ ). Alternatively, we simply counted the number of genes that had a higher coefficient of variation for the UM state compared to the gbM state; 5,357 (or 61.56\%) genes had more variable expression among 495 UM epialleles, representing a highly significant deviation from the null expectation of 50\% (binomial test, two-sided, $p$-value $\left.1.5 .10^{-4}\right)$. 


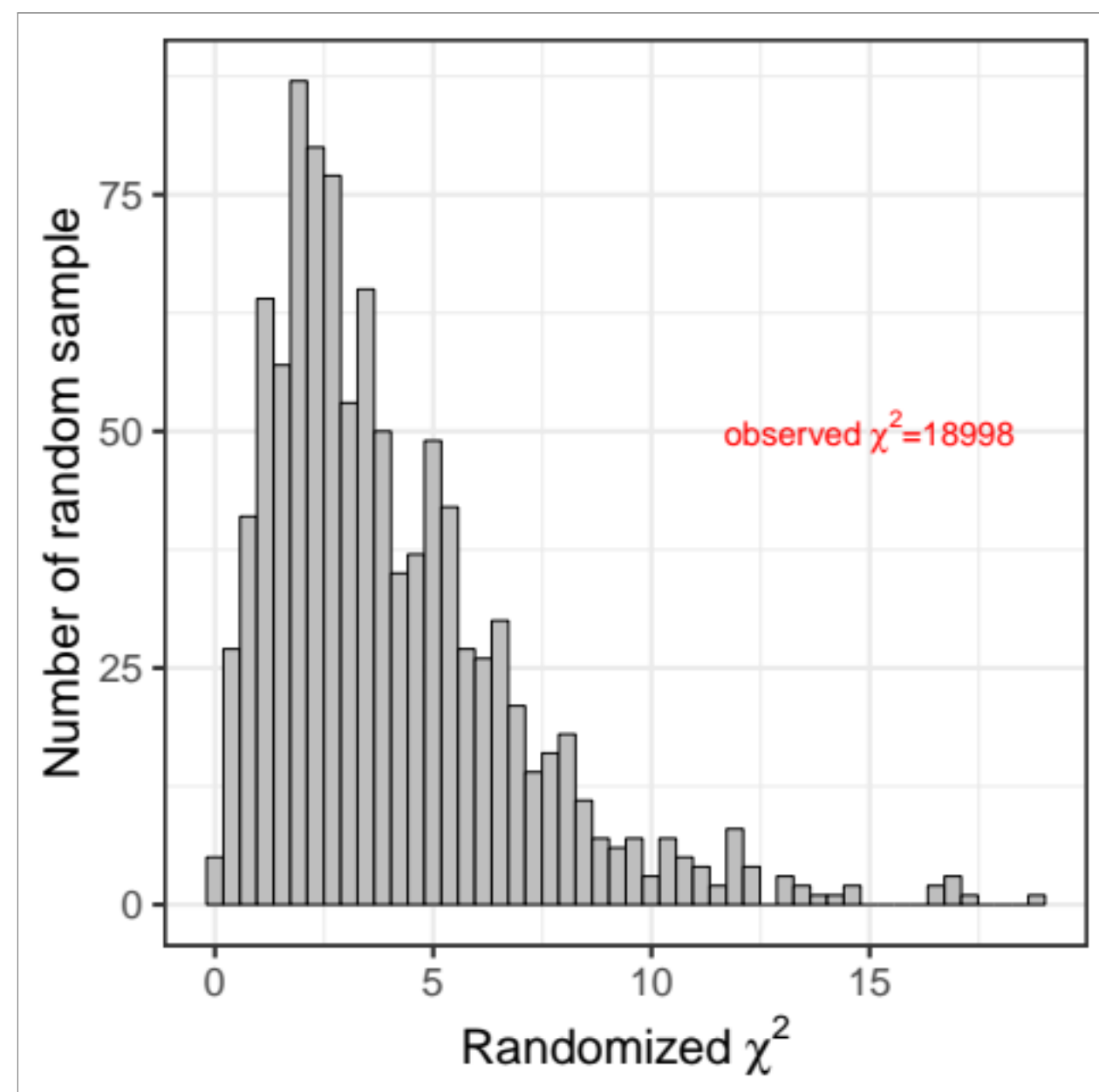

Figure 3: Distribution of $\chi^{2}$ values obtained after randomizing the data to test the association between gene methylation and expression level in the A. thaliana. For each permuted data set, a linear model with mixed effects was used to assess the correlation between methylation and expression levels (Materials and Methods, equation 5). A $\chi^{2}$ value $>7.8$ represents a significant correlation between methylation level and expression level within genes (i.e., a $p$-value $<0.05$ with 3 degrees of freedom), and such values were observed $5.4 \%$ of the time. The observed $\chi^{2}(18,998)$ was higher than the highest $\chi^{2}$ obtained on randomized data (18.94) by over a thousand-fold. 


\section{Discussion}

We have utilized the dataset of 1001 A. thaliana methylomes (Kawakatsu et al. 2016) to examine

500 features of the population genomics of epiallelic states, with a focus on gbM. Our analyses reveal two main observations. The first is that the SFS of allelic states yields information about selection. We find that all genes taken together are under selection to be UM, however, ancestrally gbM in A. thaliana are under selection to remain gbM. The second observation is that analysis of this extensive dataset has revealed an association between epiallelic state and patterns of expression, in terms of both expression

505 level and stability. Both observations have broad relevance but also have caveats that must be considered.

\section{gbM is under selection in A. thaliana:}

This study relies on 876 leaf methylomes in $A$. thaliana to investigate whether gbM is under selection

510 and also on an SFS approach to detect selection that is specifically designed for epigenomic data (Charlesworth and Jain 2014). Our work offers the first evidence that ancestrally gbM genes are subject to natural selection to remain gbM. As selection only acts on traits that impact fitness, these results suggest that gbM has a function, at least in A. thaliana and maybe in other organisms that have similar gene methylation patterns (i.e., plants, mammals and insects).

Table 1), resulting in $\gamma=4 N_{e} s_{g b M}=1.38$. Values of $4 N_{e} s$ lower than 1.0 are typically considered neutral (Charlesworth and Charlesworth 2010). The inferred values of selection coefficients acting on gbM are similar to values estimated for codon usage bias, a phenomenon known to be under weak but significant selection in species with large enough $N_{e}$ (Galtier et al. 2018). For example, leucine, valine,

520 isoleucine and arginine have estimated $\gamma$ values for selection on codon usage between 1 and 2 in $A$. 
lyrata (Qiu et al. 2011). GbM is therefore a trait that seems to have a weak impact on fitness, but natural selection may be substantial enough to maintain it in a subset of important genes through time, just like for codon bias.

Interestingly, our results on all genes taken together were opposite to those based on gbM 525 genes, because all genes are inferred to be under selection to be unmethylated (Table 1). These results suggest, first, that the selection on gbM state varies among genes and, second, that gbM might be associated with a selective trade-off (Kiefer et al. 2019). That is, gbM is advantageous for some genes, but can also be deleterious, perhaps due to the increased mutation rate on methylated cytosines, energetic costs or effects on chromatin structure. We therefore argue that the advantages of gbM 530 outweigh its putatively deleterious mutagenic effects in a subset of genes, but in other genes either gbM offers no advantage or the advantage is not strong enough to compensate for higher mutation rates.

Our inferences are of course subject to caveats. The first set of caveats is related to our application of the model of Charlesworth and Jain (2014), which assumes uniform epimutation rates across the entire genome. To help address this limitation of the model, we investigated ancestrally UM 535 and gbM genes separately, and we also separated the set of CHG-gain genes identified in E. salsugineum from the non-CHG-gain genes. We separated the latter two because CHG-gain genes may have elevated rates of de novo epimutation (Wendte et al. 2019); indeed, we estimate that CHG-gain genes have 1.77-fold higher mutation rates from the UM to gbM state. However, the datasets all inferred similar trends, including estimates of $s$ that were significantly different from zero, with

540 selection to retain methylation for ancestrally gbM genes (Table 1). These results suggest that heterogeneity in epimutation rates across genes are unlikely to drive our results, although we advocate for future investigations into CMT3 targeted genes in $A$. thaliana, because their dynamics could differ from E. salsugineum given the $\sim 47$ million year (my) divergence between species (Arias et al. 2014). Additional limitations of the model include assumptions about outcrossing, semi-dominance, 
545 independence among sites, demographic equilibrium and mutation-selection balance (Charlesworth and Jain 2014). Clearly, the first two of these assumptions are violated by our study organism (A. thaliana), which is predominantly selfing. However, we treated each individual as haploid (in the sense that we did not separate the two alleles of a gene), which reduces to sampling one allele per individual from an outcrossed population. Nonetheless, we find that the model fits the data well despite these limitations

550 (Figure 1).

Another set of limitations surround the data and our treatment of the data. For example, we used data from 10-day shoots - which are a mix of leaves, stems and leaf buds - to infer ancestral states of A. thaliana leaves. We therefore assume that shoots adequately reflect methylation states in leaves, an assumption that appears to be reasonable for genic methylation states across tissues of Brachypodium distachyon (Roessler et al. 2016). We also treated complete CDS regions as an epiallelic state - e.g., gbM, UM - and inferred the SFS of those states. We chose to employ this approach over other options, such as investigations of the per-cytosine SFS or the SFS of differentially methylated regions (DMRs) based on the study of Takuno and Gaut (2013). This study found that an ortholog that is gbM in one species is highly likely to be gbM in another species, even when the two species in question (in this

560 case, rice and Brachypodium distachyon) diverged 50 my ago. The remarkable feature of this observation is that the methylation of orthologs was conserved but the methylation of individual nucleotides was not. In other words, this and subsequent studies have suggested that gbM is a property of genes, not nucleotide sites nor DMRs, which are an amorphous and often statistically problematic concept (Roessler et al. 2016). Given their observations, Takuno and Gaut (2013) hypothesized that

$565 \mathrm{gbM}$ is a threshold character, such that the functional effects of gbM require some threshold of methylation that relies on the number and distribution of cytosines across genes. We caution that we have not explicitly tested that model here, nor inferred the properties of any threshold, but our results based on contrasting gbM and UM alleles are consistent with such a model. Our focus on genes (as 
opposed to nucleotide sites or DMRs) is also justified from observations about CHG-gain genes in $E$.

570 salsuginuem (Wendte et al. 2019).

Finally, we focus on the use of $A$. thaliana as a study organism for studies of methylation. A. thaliana has been used as a model system for good reason; without its genetic tools, the pathways and mechanisms of cytosine methylation in plants would not be nearly as well understood (Law and Jacobsen 2010). Similarly, the fact that it is selfing with a small genome size makes it ideal for some

575 applications such as population genomics and epigenomics (Alonso-Blanco et al. 2016; Kawakatsu et al. 2016), leading to the generation of unique datasets like the one we have analyzed here. However, $A$. thaliana may not be the ideal model to study methylation mutants precisely because those mutants have less phenotypic effect in A. thaliana than in some other plants - for example, methylation mutants are in maize often lethal ( $\mathrm{Li}$ et al. 2014). Consistent with this conjecture, a previous study comparing A.

580 thaliana and A. lyrata gene methylation states has inferred that $A$. thaliana has lost gbM three times faster than gaining it (Takuno et al. 2017). Our estimated values of epimutation rates on all genes (Supplementary Table S1) from gbM to UM $\left(v=2.07 .10^{-7}\right)$ and from UM to gbM $\left(\mu=6.17 .10^{-8}\right)$ exactly reiterate this three-fold difference. Thus, the growing consensus is that $A$. thaliana is losing gbM through time. We hypothesize that one reason for this is the recent shift of $A$. thaliana to an

585 inbreeding mating system, which has reduced its effective population size (Mattila et al. 2020) and likely led to weaker selection on epigenetic states. The overarching - and more important - point is that A. thaliana is likely to be a poor model to study the evolutionary forces that act on gbM, and yet our study nonetheless detects a significant selective effect.

\section{0 gbM is associated with gene expression:}

As we noted in the Introduction, the question of gbM function has been raised in many studies, and gene expression has been used as the proxy for function in most of these studies. The field has thus 
focused on a relatively simple question: Is gbM associated with gene expression? Unfortunately, the outcome of these studies has been inconsistent, owing to a wide variety of reasons that may include

595 that: i) the effect of gbM on expression is minor; ii) some studies are underpowered to detect such an effect, particularly over short temporal scales, iii) researchers disagree on statistical approaches, particularly whether UM genes can be utilized as a control comparison to gbM genes (Muyle and Gaut 2019; Bewick et al. 2019); and iv) independent epigenetic marks could have redundant functions that hide the effects of gbM loss in methylation mutants (Choi et al. 2020).

600 Our work here has, however, taken a unique approach, which is to examine the association of intraspecific variation in epialleles and expression levels across genes. This approach makes it possible to test (both within and across genes) whether a change in methylation state within the population associates with differences in expression level. To our knowledge, this is the first study to integrate intraspecific variation in methylation state with expression level in wild type plants. Our linear model consistently identified an effect of methylation state on expression, whether we investigated all of the defined states or compared pairs of states (e.g., gbM vs. UM; Table 2). The power of this approach undoubtedly comes from the extensive data generated by the 1001 methylome consortium, because the size of the estimated effect is small. In real terms, the difference between a gbM allele and a UM allele is about 1 raw sequence read, averaged over the entire data set. Nonetheless, it is clear that this result is

610 not an artifact of the approach, because we permuted the data and found that the observed results are far more extreme (by 1000-fold) than the permuted data. In short, the evidence for the effect is strong, even though it is small. This adds to a growing number of experimental and comparative genomic approaches that point consistently to some association between gbM and expression (Zilberman et al. 2007, 2008; Coleman-Derr and Zilberman 2012; Steige et al. 2017; Takuno et al. 2017; Horvath et al.

615 2019; Seymour and Gaut 2019). We also show that the variation in gene expression among accessions is lower for the gbM compared to the UM epiallelic state. This is in agreement with other studies that 
suggest that gbM stabilizes gene expression (Zilberman et al. 2008; Coleman-Derr and Zilberman 2012; Steige et al. 2017; Takuno et al. 2017; Horvath et al. 2019; Seymour and Gaut 2019).

Our results point to selection on gbM perhaps, in part, due to its association with gene

620 expression. But there remain two difficult questions. The first is whether selection is on gbM itself i.e., the epigenetic states directly - or on associated factors, such as chromatin factors or even underlying sequence features that may contribute to gbM in some unknown way. Unfortunately, we find no convincing method to discriminate among an associated versus a direct effect of gbM, and we must thus be careful to conclude that selection acts directly on the epigenetic state. However, to

625 investigate this question, we ran a linear model with mixed effects to study the association between the number of CG dinucleotides (\#CG) and gene methylation states in the Salk Institute data (see Materials and Methods for details). There was a significant correlation between \#CG and methylation states $\left(\chi^{2}=\right.$ 17,262 and $p$-value $=0$ when comparing a linear model with and without gene methylation state effect). This model also demonstrates that gbM epialleles have more CG dinucleotides than UM epialleles

630 (linear model pairwise contrast estimate $=1.338, \mathrm{p}<0.0001$ ). Surprisingly, however, when including both methylation state and \#CG in a linear model to explain expression variation (see Materials and Methods), the methylation state remains the main influence on gene expression. Moreover, accessions with higher \#CG are significantly less expressed (linear model estimate $-5.77 .10^{-3}, \mathrm{p}<2 \mathrm{e}-16$ ), which opposes the effect of gbM on expression. Together, these analyses illustrate that the epiallelic state is 635 not independent of the underlying sequence, as measured by \#CG, but it also hints that epigenetic state contributes to phenotype in a way that is not easily explained by variation in the number of CG dinucleotides alone.

The second difficult question is function: what does gbM actually do? We cannot yet answer this question, especially given the inconsistent evidence from a variety of organisms and experiments 640 (Zilberman et al. 2007, 2008; Coleman-Derr and Zilberman 2012; Li-Byarlay et al. 2013; Yearim et al. 
2015; Bewick et al. 2016, 2019; Neri et al. 2017; Steige et al. 2017; Teissandier and Bourc'his 2017;

Takuno et al. 2017; Horvath et al. 2019; Muyle and Gaut 2019; Seymour and Gaut 2019; Choi et al. 2020). We note, however, that histone H1 was recently shown to have a similar effect to DNA methylation in TEs and genes (Choi et al. 2020). In that study, expression of antisense transcripts was

645 activated in 710 genes following methylation loss in h1,met1 double mutants, at a level that was not positively correlated to sense transcription changes. This finding definitely demonstrates that, at least for some genes, gbM can repress antisense transcription in A. thaliana jointly with $\mathrm{H} 1$. We hypothesize that the inhibition of antisense transcription requires a threshold of cytosine methylation, which we have captured by studying the methylation state for the entire CDS. Even if this is true, there are still

650 unanswered mechanistic questions about how the effect of gbM on anti-sense transcription affects the level and stability of expression.

\section{Authors contributions}

655 AM and BSG conceived the project. AM ran the analyses. JRI provided the MCMC R code and critical ideas. DKS shared data. AM and BSG wrote the manuscript with input from all authors.

\section{Acknowledgements}

AM is supported by an EMBO Postdoctoral Fellowship ALTF 775-2017 and by HFSPO Fellowship

660 LT000496/2018-L. BSG is supported by NSF grant IOS-1542703. JRI is supported by NSF grant 1546719 and USDA Hatch project CA-D-PLS-2066-H. We would like to thank Mike May for help developing the MCMC approach used here. 
bioRxiv preprint doi: https://doi.org/10.1101/2020.09.04.283333; this version posted September 4, 2020. The copyright holder for this preprint (which was not certified by peer review) is the author/funder, who has granted bioRxiv a license to display the preprint in perpetuity. It is made available under aCC-BY-NC-ND 4.0 International license.

\section{Data Availability}

All data used in this manuscript were previously published (GEO accessions GSE43857, GSE80744, GSE54292, GSE43858, GSE54680).

\section{References}

Alonso-Blanco C., J. Andrade, C. Becker, F. Bemm, J. Bergelson, et al., 2016 1,135 Genomes Reveal the Global Pattern of Polymorphism in Arabidopsis thaliana. Cell 166: 481-491. https://doi.org/ 10.1016/j.cell.2016.05.063

Arias T., M. A. Beilstein, M. Tang, M. R. McKain, and J. C. Pires, 2014 Diversification times among Brassica (Brassicaceae) crops suggest hybrid formation after 20 million years of divergence. Am. J. Bot. 101: 86-91. https://doi.org/10.3732/ajb.1300312

Bates D., M. Mächler, B. Bolker, and S. Walker, 2015 Fitting Linear Mixed-Effects Models Using lme4. Journal of Statistical Software. https://doi.org/10.18637/jss.v067.i01

Becker C., J. Hagmann, J. Müller, D. Koenig, O. Stegle, et al., 2011 Spontaneous epigenetic variation in the Arabidopsis thaliana methylome. Nature 480: 245-249. https://doi.org/10.1038/nature10555

Bewick A. J., L. Ji, C. E. Niederhuth, E.-M. Willing, B. T. Hofmeister, et al., 2016 On the origin and evolutionary consequences of gene body DNA methylation. PNAS 113: 9111-9116. https://doi.org/10.1073/pnas.1604666113

Bewick A. J., and R. J. Schmitz, 2017 Gene body DNA methylation in plants. Curr. Opin. Plant Biol. 36: 103-110. https://doi.org/10.1016/j.pbi.2016.12.007

Bewick A. J., Y. Zhang, J. M. Wendte, X. Zhang, and R. J. Schmitz, 2019 Evolutionary and Experimental Loss of Gene Body Methylation and Its Consequence to Gene Expression. G3 (Bethesda). https://doi.org/10.1534/g3.119.400365

Bird A. P., 1980 DNA methylation and the frequency of CpG in animal DNA. Nucleic Acids Res. 8: 1499-1504. https://doi.org/10.1093/nar/8.7.1499 
Boukas L., H. T. Bjornsson, and K. D. Hansen, 2020 Purifying selection acts on germline methylation to modify the CpG mutation rate at promoters. bioRxiv 2020.07.04.187880.

https://doi.org/10.1101/2020.07.04.187880

Charlesworth B., and D. Charlesworth, 2010 Elements of Evolutionary Genetics. Roberts and Company Publishers.

Charlesworth B., and K. Jain, 2014 Purifying selection, drift, and reversible mutation with arbitrarily high mutation rates. Genetics 198: 1587-1602. https://doi.org/10.1534/genetics.114.167973

Choi J., D. B. Lyons, M. Y. Kim, J. D. Moore, and D. Zilberman, 2020 DNA Methylation and Histone H1 Jointly Repress Transposable Elements and Aberrant Intragenic Transcripts. Mol. Cell 77: 310-323.e7. https://doi.org/10.1016/j.molcel.2019.10.011

Cokus S. J., S. Feng, X. Zhang, Z. Chen, B. Merriman, et al., 2008 Shotgun bisulphite sequencing of the Arabidopsis genome reveals DNA methylation patterning. Nature 452: 215-219. https://doi.org/10.1038/nature06745

Coleman-Derr D., and D. Zilberman, 2012 DNA methylation, H2A.Z, and the regulation of constitutive expression. Cold Spring Harb. Symp. Quant. Biol. 77: 147-154.

https://doi.org/10.1101/sqb.2012.77.014944

Dubin M. J., P. Zhang, D. Meng, M.-S. Remigereau, E. J. Osborne, et al., 2015 DNA methylation in Arabidopsis has a genetic basis and shows evidence of local adaptation. Elife 4: e05255. https:// doi.org/10.7554/eLife.05255

Galtier N., C. Roux, M. Rousselle, J. Romiguier, E. Figuet, et al., 2018 Codon Usage Bias in Animals: Disentangling the Effects of Natural Selection, Effective Population Size, and GC-Biased Gene Conversion. Mol. Biol. Evol. 35: 1092-1103. https://doi.org/10.1093/molbev/msy015

Graaf A. van der, R. Wardenaar, D. A. Neumann, A. Taudt, R. G. Shaw, et al., 2015 Rate, spectrum, and evolutionary dynamics of spontaneous epimutations. Proc. Natl. Acad. Sci. U.S.A. 112: 66766681. https://doi.org/10.1073/pnas.1424254112 
Hernandez R. D., S. H. Williamson, L. Zhu, and C. D. Bustamante, 2007 Context-dependent mutation rates may cause spurious signatures of a fixation bias favoring higher GC-content in humans. Mol. Biol. Evol. 24: 2196-2202. https://doi.org/10.1093/molbev/msm149

Horvath R., B. Laenen, S. Takuno, and T. Slotte, 2019 Single-cell expression noise and gene-body methylation in Arabidopsis thaliana. Heredity (Edinb) 123: 81-91. https://doi.org/10.1038/s41437-018-0181-z

Kawakatsu T., S.-S. C. Huang, F. Jupe, E. Sasaki, R. J. Schmitz, et al., 2016 Epigenomic Diversity in a Global Collection of Arabidopsis thaliana Accessions. Cell 166: 492-505. https://doi.org/10.1016/j.cell.2016.06.044

Kawashima T., and F. Berger, 2014 Epigenetic reprogramming in plant sexual reproduction. Nat. Rev. Genet. 15: 613-624. https://doi.org/10.1038/nrg3685

Kiefer C., E.-M. Willing, W.-B. Jiao, H. Sun, M. Piednoël, et al., 2019 Interspecies association mapping links reduced CG to TG substitution rates to the loss of gene-body methylation. Nat Plants 5: 846-855. https://doi.org/10.1038/s41477-019-0486-9

Korunes K. L., and K. Samuk, 2020 pixy: Unbiased estimation of nucleotide diversity and divergence in the presence of missing data. bioRxiv 2020.06.27.175091. https://doi.org/10.1101/2020.06.27.175091

Law J. A., and S. E. Jacobsen, 2010 Establishing, maintaining and modifying DNA methylation patterns in plants and animals. Nat. Rev. Genet. 11: 204-220. https://doi.org/10.1038/nrg2719

Lenth R. V., 2016 Least-Squares Means: The R Package lsmeans. Journal of Statistical Software 69: 133. https://doi.org/10.18637/jss.v069.i01

Li Q., S. R. Eichten, P. J. Hermanson, V. M. Zaunbrecher, J. Song, et al., 2014 Genetic perturbation of the maize methylome. Plant Cell 26: 4602-4616. https://doi.org/10.1105/tpc.114.133140

Li-Byarlay H., Y. Li, H. Stroud, S. Feng, T. C. Newman, et al., 2013 RNA interference knockdown of DNA methyl-transferase 3 affects gene alternative splicing in the honey bee. PNAS 110: 12750-12755. https://doi.org/10.1073/pnas.1310735110 
Lister R., R. C. O’Malley, J. Tonti-Filippini, B. D. Gregory, C. C. Berry, et al., 2008 Highly integrated single-base resolution maps of the epigenome in Arabidopsis. Cell 133: 523-536. https://doi.org/10.1016/j.cell.2008.03.029

Luo C., P. Hajkova, and J. R. Ecker, 2018 Dynamic DNA methylation: In the right place at the right time. Science 361: 1336-1340. https://doi.org/10.1126/science.aat6806

Lyons E., and M. Freeling, 2008 How to usefully compare homologous plant genes and chromosomes as DNA sequences. Plant J. 53: 661-673. https://doi.org/10.1111/j.1365-313X.2007.03326.X

Mattila T. M., B. Laenen, and T. Slotte, 2020 Population Genomics of Transitions to Selfing in Brassicaceae Model Systems. Methods Mol. Biol. 2090: 269-287. https://doi.org/10.1007/9781-0716-0199-0_11

Maunakea A. K., R. P. Nagarajan, M. Bilenky, T. J. Ballinger, C. D’Souza, et al., 2010 Conserved role of intragenic DNA methylation in regulating alternative promoters. Nature 466: 253-257. https://doi.org/10.1038/nature09165

Miura A., M. Nakamura, S. Inagaki, A. Kobayashi, H. Saze, et al., 2009 An Arabidopsis jmjC domain protein protects transcribed genes from DNA methylation at CHG sites. EMBO J. 28: 10781086. https://doi.org/10.1038/emboj.2009.59

Muyle A., and B. S. Gaut, 2019 Loss of Gene Body Methylation in Eutrema salsugineum Is Associated with Reduced Gene Expression. Mol. Biol. Evol. 36: 155-158. https://doi.org/10.1093/molbev/ msy204

Neri F., S. Rapelli, A. Krepelova, D. Incarnato, C. Parlato, et al., 2017 Intragenic DNA methylation prevents spurious transcription initiation. Nature 543: 72-77. https://doi.org/10.1038/nature21373

Niederhuth C. E., A. J. Bewick, L. Ji, M. S. Alabady, K. D. Kim, et al., 2016 Widespread natural variation of DNA methylation within angiosperms. Genome Biology 17: 194. https://doi.org/10.1186/s13059-016-1059-0

Nordborg M., T. T. Hu, Y. Ishino, J. Jhaveri, C. Toomajian, et al., 2005 The Pattern of Polymorphism in Arabidopsis thaliana. PLoS Biology 3: e196. https://doi.org/10.1371/journal.pbio.0030196 
Ossowski S., K. Schneeberger, J. I. Lucas-Lledó, N. Warthmann, R. M. Clark, et al., 2010 The rate and molecular spectrum of spontaneous mutations in Arabidopsis thaliana. Science 327: 92-94. https://doi.org/10.1126/science.1180677

Qiu S., K. Zeng, T. Slotte, S. Wright, and D. Charlesworth, 2011 Reduced Efficacy of Natural Selection on Codon Usage Bias in Selfing Arabidopsis and Capsella Species. Genome Biology and Evolution 3: 868-880. https://doi.org/10.1093/gbe/evr085

Reinders J., B. B. H. Wulff, M. Mirouze, A. Marí-Ordóñez, M. Dapp, et al., 2009 Compromised stability of DNA methylation and transposon immobilization in mosaic Arabidopsis epigenomes. Genes Dev. 23: 939-950. https://doi.org/10.1101/gad.524609

Roessler K., S. Takuno, and B. S. Gaut, 2016 CG Methylation Covaries with Differential Gene Expression between Leaf and Floral Bud Tissues of Brachypodium distachyon. PLOS ONE 11: e0150002. https://doi.org/10.1371/journal.pone.0150002

Saze H., A. Shiraishi, A. Miura, and T. Kakutani, 2008 Control of genic DNA methylation by a jmjC domain-containing protein in Arabidopsis thaliana. Science 319: 462-465. https://doi.org/10.1126/science.1150987

Schmitz R. J., M. D. Schultz, M. G. Lewsey, R. C. O’Malley, M. A. Urich, et al., 2011 Transgenerational epigenetic instability is a source of novel methylation variants. Science 334: 369-373. https://doi.org/10.1126/science.1212959

Schmitz R. J., Z. A. Lewis, and M. G. Goll, 2019 DNA Methylation: Shared and Divergent Features across Eukaryotes. Trends Genet. 35: 818-827. https://doi.org/10.1016/j.tig.2019.07.007

Seymour D. K., D. Koenig, J. Hagmann, C. Becker, and D. Weigel, 2014 Evolution of DNA methylation patterns in the Brassicaceae is driven by differences in genome organization. PLoS Genet. 10: e1004785. https://doi.org/10.1371/journal.pgen.1004785

Seymour D. K., and B. S. Gaut, 2019 Phylogenetic shifts in gene body methylation correlate with gene expression and reflect trait conservation. Mol. Biol. Evol. https://doi.org/10.1093/molbev/msz195 
Steige K. A., B. Laenen, J. Reimegård, D. G. Scofield, and T. Slotte, 2017 Genomic analysis reveals major determinants of cis-regulatory variation in Capsella grandiflora. Proc. Natl. Acad. Sci. U.S.A. 114: 1087-1092. https://doi.org/10.1073/pnas.1612561114

Stroud H., M. V. C. Greenberg, S. Feng, Y. V. Bernatavichute, and S. E. Jacobsen, 2013 Comprehensive analysis of silencing mutants reveals complex regulation of the Arabidopsis methylome. Cell 152: 352-364. https://doi.org/10.1016/j.cell.2012.10.054

Takuno S., and B. S. Gaut, 2012 Body-methylated genes in Arabidopsis thaliana are functionally important and evolve slowly. Mol. Biol. Evol. 29: 219-227. https://doi.org/10.1093/molbev/msr188

Takuno S., and B. S. Gaut, 2013 Gene body methylation is conserved between plant orthologs and is of evolutionary consequence. Proc. Natl. Acad. Sci. U.S.A. 110: 1797-1802. https://doi.org/10.1073/pnas.1215380110

Takuno S., J.-H. Ran, and B. S. Gaut, 2016 Evolutionary patterns of genic DNA methylation vary across land plants. Nat Plants 2: 15222. https://doi.org/10.1038/nplants.2015.222

Takuno S., D. K. Seymour, and B. S. Gaut, 2017 The Evolutionary Dynamics of Orthologs That Shift in Gene Body Methylation between Arabidopsis Species. Mol. Biol. Evol. 34: 1479-1491. https://doi.org/10.1093/molbev/msx099

Teissandier A., and D. Bourc'his, 2017 Gene body DNA methylation conspires with H3K36me3 to preclude aberrant transcription. EMBO J. 36: 1471-1473. https://doi.org/10.15252/embj.201796812

Teixeira F. K., and V. Colot, 2009 Gene body DNA methylation in plants: a means to an end or an end to a means? EMBO J. 28: 997-998. https://doi.org/10.1038/emboj.2009.87

Tran R. K., J. G. Henikoff, D. Zilberman, R. F. Ditt, S. E. Jacobsen, et al., 2005 DNA methylation profiling identifies CG methylation clusters in Arabidopsis genes. Curr. Biol. 15: 154-159. https://doi.org/10.1016/j.cub.2005.01.008

Vidalis A., D. Živković, R. Wardenaar, D. Roquis, A. Tellier, et al., 2016 Methylome evolution in plants. Genome Biol. 17: 264. https://doi.org/10.1186/s13059-016-1127-5 
Wang J., and C. Fan, 2014 A neutrality test for detecting selection on DNA methylation using single methylation polymorphism frequency spectrum. Genome Biol Evol 7: 154-171. https://doi.org/ 10.1093/gbe/evu271

Wendte J. M., Y. Zhang, L. Ji, X. Shi, R. R. Hazarika, et al., 2019 Epimutations are associated with CHROMOMETHYLASE 3-induced de novo DNA methylation. Elife 8. https://doi.org/10.7554/eLife.47891

Xu G., J. Lyu, Q. Li, H. Liu, D. Wang, et al., 2020 Adaptive evolution of DNA methylation reshaped gene regulation in maize. bioRxiv 2020.03.13.991117.

https://doi.org/10.1101/2020.03.13.991117

Yearim A., S. Gelfman, R. Shayevitch, S. Melcer, O. Glaich, et al., 2015 HP1 Is Involved in Regulating the Global Impact of DNA Methylation on Alternative Splicing. Cell Reports 10: 1122-1134. https://doi.org/10.1016/j.celrep.2015.01.038

Zhang X., J. Yazaki, A. Sundaresan, S. Cokus, S. W.-L. Chan, et al., 2006 Genome-wide highresolution mapping and functional analysis of DNA methylation in arabidopsis. Cell 126: 1189_ 1201. https://doi.org/10.1016/j.cell.2006.08.003

Zilberman D., M. Gehring, R. K. Tran, T. Ballinger, and S. Henikoff, 2007 Genome-wide analysis of Arabidopsis thaliana DNA methylation uncovers an interdependence between methylation and transcription. Nat. Genet. 39: 61-69. https://doi.org/10.1038/ng1929

Zilberman D., D. Coleman-Derr, T. Ballinger, and S. Henikoff, 2008 Histone H2A.Z and DNA methylation are mutually antagonistic chromatin marks. Nature 456: 125-129. https://doi.org/10.1038/nature07324

Zilberman D., 2017 An evolutionary case for functional gene body methylation in plants and animals. Genome Biol. 18: 87. https://doi.org/10.1186/s13059-017-1230-2 